\title{
The TWINS exospheric neutral H-density distribution under solar minimum conditions
}

\author{
J. H. Zoennchen ${ }^{1}$, J. J. Bailey ${ }^{2}$, U. Nass ${ }^{1}$, M. Gruntman ${ }^{2}$, H. J. Fahr ${ }^{1}$, and J. Goldstein ${ }^{3,4}$ \\ ${ }^{1}$ Argelander Institut für Astronomie, Astrophysics Department, University of Bonn, Auf dem Huegel 71, 53121 Bonn, \\ Germany \\ ${ }^{2}$ University of Southern California, Department of Astronautical Engineering, Viterbi School of Engineering, Los Angeles, \\ CA 90089-1192, USA \\ ${ }^{3}$ Southwest Research Institute, San Antonio, TX, USA \\ ${ }^{4}$ University of Texas, San Antonio, San Antonio, TX, USA
}

Received: 13 July 2011 - Revised: 29 September 2011 - Accepted: 28 November 2011 - Published: 1 December 2011

\begin{abstract}
Terrestrial exospheric atomic hydrogen $(\mathrm{H})$ resonantly scatters solar Lyman- $\alpha(121.567 \mathrm{~nm})$ radiation, observed as the glow of the H-geocorona. The Two Wide-angle Imaging Neutral-atom Spectrometers (TWINS) satellites are equiped with two Lyman- $\alpha$ line-of-sight Detectors (LADs) each. Since during the past solar minimum conditions the relevant solar control parameters practically did not vary, we are using LAD data between June and September 2008 to create a time averaged hydrogen geocorona model representative for these solar minimum conditions. In this averaged model we assume that the $\mathrm{H}$-geocorona is longitudinally symmetric with respect to the earth-sun line. We find a 3-dimensional $\mathrm{H}$-density distribution in the range from 3 to 8 earth radii which with some caution can also be extrapolated to larger distances. For lower geocentric distances than 3 earth radii a best fitting r-dependent Chamberlain (1963)-like model is adapted. Main findings are larger than conventionally expected H-densities at heights above $5 R_{\mathrm{E}}$ and a pronounced day-to-night side $\mathrm{H}$-density asymmetry. The H-geocorona presented here should serve as a reference $\mathrm{H}$-atmosphere for the earth during solar minimum conditions.
\end{abstract}

Keywords. Atmospheric composition and structure (Airglow and aurora; Pressure, density, and temperature) - Meteorology and atmospheric dynamics (Thermospheric dynamics)

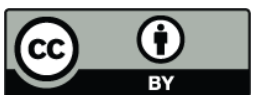

Correspondence to: $\mathrm{J}$. H. Zoennchen (zoenn@astro.uni-bonn.de)

\section{Introduction}

The atomic hydrogen $(\mathrm{H})$ in Earth's upper exosphere resonantly scatters solar Lyman- $\alpha(121.567 \mathrm{~nm})$ radiation, creating the ultraviolet optical signature known as the Lyman- $\alpha$ geocorona (Kupperian et al., 1959; Carruthers et al., 1976). Since its earliest measurement by rocket-based instruments, numerous models have been formulated to provide estimates of the neutral exospheric $\mathrm{H}$ density (e.g. Johnson, 1961; Chamberlain, 1963; Thomas and Bohlin, 1972; Fahr and Shizgal, 1983; Rairden et al., 1986; Bishop, 1991; Hodges, 1994; Østgaard et al., 2003).

Two Wide-angle Imaging Neutral-atom Spectrometers (TWINS) (McComas et al., 2009) is the first stereoscopic magnetospheric energetic neutral atom (ENA) imaging mission. The TWINS satellites perform simultaneous imaging from two separate spacecraft on widely-separated Molniya orbits. Its ENA imagers observe neutrals produced via charge exchange between magnetospheric ions and exospheric neutrals. Extracting ion density distribution from ENA images requires knowledge of the neutral exospheric $\mathrm{H}$ density. For this reason, TWINS instrumentation also includes detectors that register geocoronal ultraviolet light which can be used to quantify the extended neutral $\mathrm{H}$ distribution.

Each TWINS spacecraft includes a pair of Lyman- $\alpha$ sensitive broadband photometers, Lyman- $\alpha$ Detectors (LADs), centered at the wavelength $122 \mathrm{~nm}$ with a bandwidth of $10 \mathrm{~nm}$, to register line-of-sight (LOS) integrated resonantly scattered Lyman- $\alpha$ intensities of the H-geocorona. Zoennchen (2006) and Nass et al. (2006) proposed specifically for LAD data analysis a simplification of the 144-coefficient model from Hodges (1994); the simplified version needs only 12 coefficients. Using this model formulation, the first

Published by Copernicus Publications on behalf of the European Geosciences Union. 
analysis of TWINS geocoronal data (Zoennchen et al., 2010) provided model $\mathrm{H}$ densities for three specific two-day periods during deep quiet conditions. In this paper we use TWINS1-LAD measurements to derive an averaged global $\mathrm{H}$ atom distribution using LAD data from June to September 2008. The derived H-density distribution is intended for use during all solar quiet conditions from June 2008 through early 2010. This averaged model, which is based upon the parameterizations already performed for specific case studies (Zoennchen, 2006; Zoennchen et al., 2010; Bailey and Gruntman, 2011), is an advancement in contrast to the commonly used Rairden et al. (1986) model, in that it introduces important asymmetries that impact the results of extracting ion densities from ENA images through inversion or forward modeling (e.g. Grimes et al., 2010).

\section{Approach}

The LAD measured intensity is the sum of the geocoronal and interplanetary glow. We subtract the interplanetary glow using a representative all-sky map based on SWAN/SOHO measurements (Bertaux et al., 1997). The interplanetary glow usually accounts for $10-50$ percent of the LAD measured intensities.

The extension of the neutral H-geocorona strongly depends on solar activity. Since there were nearly constant solar (quiet) conditions from June to September 2008 it is reasonable to construct an average quiet-time H-density model using TWINS Lyman- $\alpha$ data from this entire three month period. This model fit is assumed to represent the approximately time-invariant neutral exospheric $\mathrm{H}$ density distribution under solar minimum conditions. Because such a formulation cannot capture seasonal variations caused by the change of the Earth's polar-zenith angle, and for utility in magnetospheric ion density retrieval, the parameterization uses the GSE coordinate system.

A description of the resonant Lyman- $\alpha$ scattering process within the terrestrial neutral exosphere, the method of enfolding the line of sight integrated TWINS1-LAD data into a 3-D neutral $\mathrm{H}$ density distribution and details about the TWINSLAD instrument can be found in Zoennchen (2006); Zoennchen et al. (2010); Bailey and Gruntman (2011).

\section{Observational coverage}

Good observational coverage of circumterrestrial space is essential for obtaining reliable exospheric $\mathrm{H}$ distributions because the global fitting is constrained by regions with actual measurements. In general, the TWINS observational geometry provides excellent coverage of the Northern Hemisphere, where the orbit apogees are located, but may be limited in the Southern Hemisphere. Observational coverage is further limited by the sun angle, i.e. the orbit's orientation with respect to the seasonal position of the Sun. To avoid possible solar contamination, only those measurements with a detector LOS pointed $>90^{\circ}$ from the direction to the Sun are used. Starting in early August 2008, the TWINS1-LAD dayside lines-of-sight became oriented $<90^{\circ}$ from the solar direction and therefore had to be excluded.

Our model distribution incorporates geocoronal measurements from geocentric distances in the range $3-8 R_{\mathrm{E}}$. During each highly-elliptical orbit of the TWINS1 satellite, the geocentric distance of where the LAD instruments LOS makes its closest approach to the Earth (=Earth intersection distance $\left.r_{\text {E.i.d. }}\right)$ varies from 2.0-6.5 Earth radii $\left(R_{\mathrm{E}}\right)$. Below $3 R_{\mathrm{E}}$, the geocorona deviates substantially from an optically thin condition. Thus, measurements with $r_{\text {E.i.d. }}<3 R_{\mathrm{E}}$ are excluded from our present analysis. We also exclude measurements in which the LOS passes through Earth's shadow, approximated by a cylinder (with $1 R_{\mathrm{E}}$ radius) in the antisolar direction. Intrinsic uncertainty in the knowledge of interplanetary glow makes the prediction of $\mathrm{H}$ densities at very large distances less reliable.

\section{Coordinate system}

The model fit of the neutral H-geocorona uses standard GSEcoordinates. The X-Y-plane is equal to the ecliptical plane, the $\mathrm{Z}$-axis points towards the ecliptic north pole. The geocentric distance $r$ is in $R_{\mathrm{E}}$. The longitudinal angle $\phi$ is counted from $0^{\circ}$ (sun direction) counter clockwise to $360^{\circ}$ (with $180^{\circ}$ at the antisolar direction). The latitudinal angle $\theta$ is counted from the $\mathrm{Z}$-axis $\left(0^{\circ}\right.$ at the ecliptic north pole) to $180^{\circ}$ (at the ecliptic south pole).

\section{Data selection and processing}

To obtain a representative dataset of the solar quiet time between June and September 2008, we selected TWINS1-LAD data of 4 different time sections, namely of 11 June, 2224 July, 15-17 August and 1-13 September 2008. Every section provides the same amount of valid measurements in order to enforce an equal contribution of each section to the final result. All fitted data passed a data cleaning process before they were used to make sure that invalid data (e.g. caused by reflections, earth shadow passings, invalid spacecraft operations) are ruled out. In addition all lines of sight with earth intersection distances closer than $3 R_{\mathrm{E}}$ are thus removed for fullfilling the optically thin requirement of the used single scattering approximation. Figure 1 shows the measured Lyman- $\alpha$ intensities versus their geocentric earth intersection distance for all used data with a separate color for every time section.

Following Pryor et al. (1998) we obtained the solar Lyman- $\alpha$ line centered flux from the total solar Lyman- $\alpha$ flux as taken from SORCE SOLSTICE (see Sect. 6 and Fig. 2) adjusted by a factor of 0.9 . The data correction from the contribution of Lyman- $\alpha$ background sources other than 


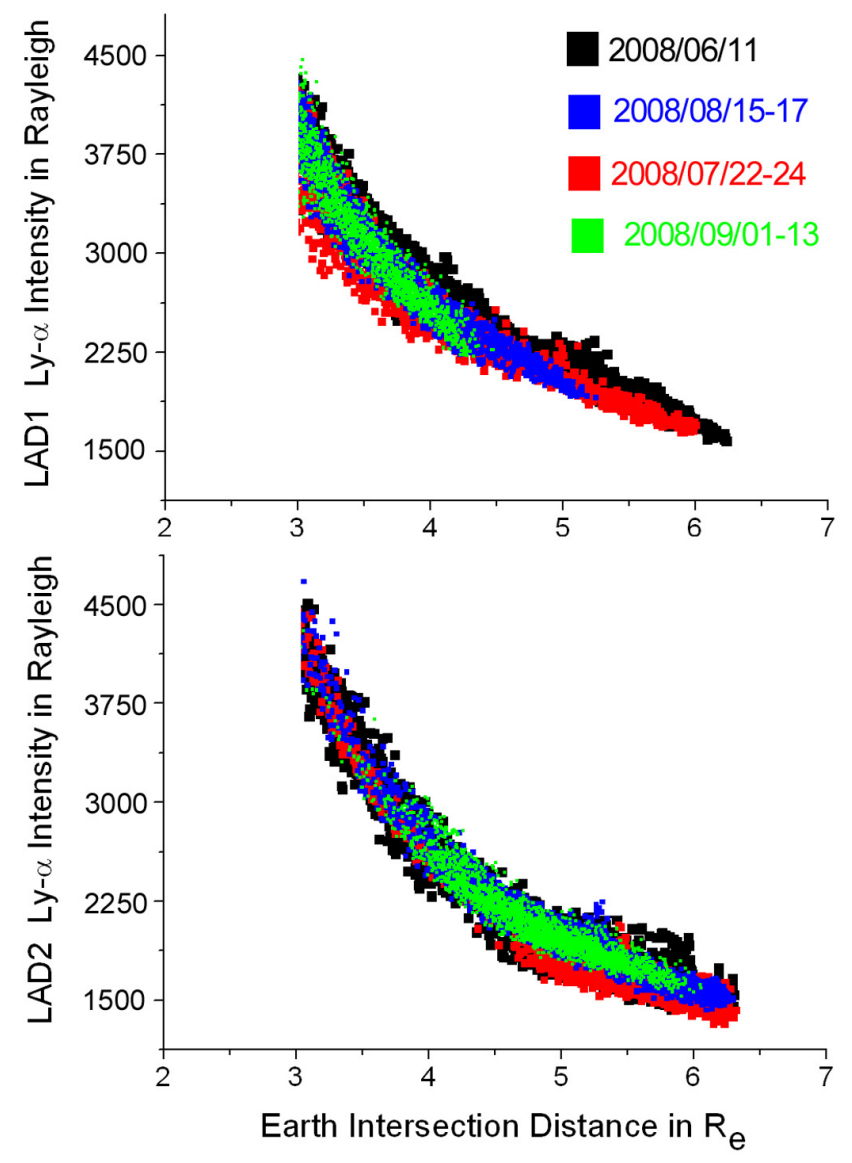

Fig. 1. The used TWINS1 LAD1 (upper section) and LAD2 (lower section) Lyman- $\alpha$ intensity data (uncleaned) versus their geocentric earth intersection distance (black $=11$ June, red=22-24 July, blue $=15-17$ August, green $=1-13$ September).

the interplanetary medium (e.g. earth albedo Lyman- $\alpha$ glow, bright stars and transcharged fast solar $\mathrm{H}$-atoms) was done in an equal manner as detailed described already in Zoennchen et al. (2010). The correction of the interplanetary Lyman- $\alpha$ background radiation is described in Sect. 7.

\section{Solar conditions}

From 11 June to 13 September 2008 as the relevant time period of this analysis the solar activity conditions were quite stable and at an extraordinary low level (see Fig. 2).

The solar $10.7 \mathrm{~cm}$ radioflux, which is an index for the solar activity and correlates to some degree with the solar Lyman$\alpha$ radiation output, fluctuated only by about $\pm 2.5 \%$ around its average value of $\approx 68\left[10^{-22} \mathrm{~W} \mathrm{~m}^{-2} \mathrm{~Hz}^{-1}\right]$ in the mentioned time period (see Fig. 2, lower black curve). At the same time the composite total solar Lyman- $\alpha$ flux measured by TIMED SEE and SORCE SOLSTICE calibrated to UARS SOLSTICE level (Woods et al., 2000) (provided by LASP, Laboratory For Atmospheric And Space Physics, University

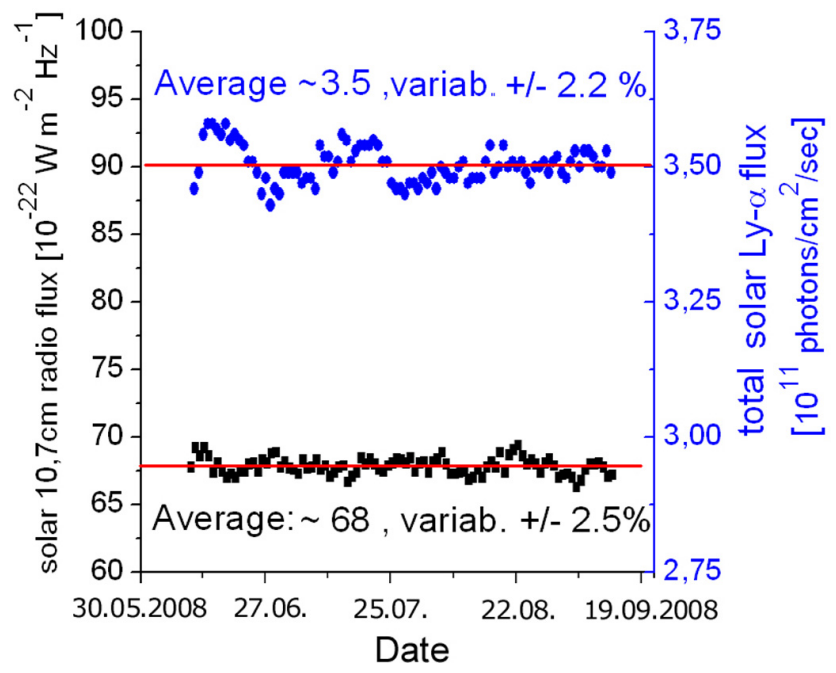

Fig. 2. The composite, total solar Lyman- $\alpha$ flux taken from TIMED SEE and SORCE SOLSTICE (upper blue graph) and the terrestrial solar $10.7 \mathrm{~cm}$ radioflux taken from NGDC (lower black graph) both show a very low variability of $2.2-2.5 \%$ in the relevant time section between 11 June and 13 September 2008.

of Boulder, Colorado) has fluctuations of $\pm 2.2 \%$ around its average value of $3.5\left[10^{11}\right.$ photons $\left.\mathrm{cm}^{-2} \mathrm{~s}^{-1}\right]$ (see Fig. 2, upper blue curve). Those nearly invariable solar activity parameters in the mentioned time period allow the assumptions of stable, invariant geocoronal resonant scattering conditions and a nearly constant interplanetary Lyman- $\alpha$ backscattering glow (see Sect. 7) in these data analysis.

\section{Interplanetary Lyman- $\alpha$-background radiation cor- rection}

The interplanetary Lyman- $\alpha$ glow which is originating from resonant backscattering of solar Lyman- $\alpha$ radiation by $\mathrm{H}$ atoms of the neutral interplanetary medium needs to be subtracted as a background source from the TWINS1-LAD data. To do this quantitative correction we use an all-sky-map of the interplanetary Lyman- $\alpha$ radiation (see Fig. 3). This map is essentially based on a model fit done by W. Pryor and R. Gladstone (private communication, 2011) with the use of 11 June 2008-data from the SOHO/SWAN instrument (Bertaux et al., 1997) and contains larger (but more realistic) intensities, than used in Zoennchen et al. (2010). A more detailed description of fitting the interplanetary Lyman$\alpha$ glow can be also found in Pryor et al. (1992) and Pryor et al. (2008).

Very small fluctuations of the solar (i.e. interplanetary) Lyman- $\alpha$-radiation from June to September 2008 associated with tiny changes of the solar activity and the parallax effect caused by the earth motion around the sun during the relevant 3 months contribute with an additional systematic error. To investigate these errors introduced by using a single 


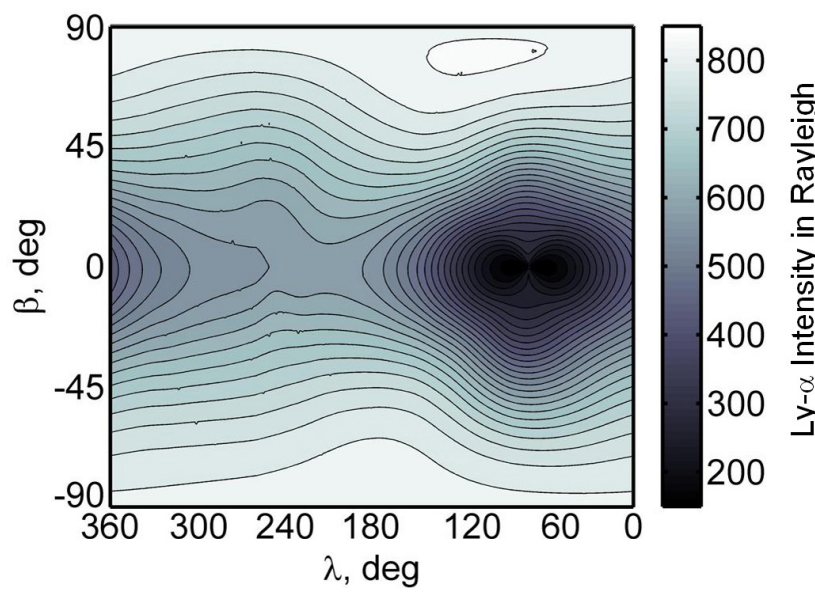

Fig. 3. Interplanetary Lyman- $\alpha$ glow intensity all-sky-map of 11 June 2008 derived from SOHO/SWAN-data and solar anisotropy measurements (W. Pryor and R. Gladstone, private comunication, 2011) in geocentric, ecliptical coordinates.

representative all-sky map for the June to September 2008 time period, we compared interplanetary glow maps provided by W. Pryor and R. Gladstone (private communication, 2011) for the first and last day used in the fitting procedure. The TWINS1-LAD observed regions differed by an average of $5.6 \%$ of the actual observed intensities.

\section{Model}

Since the geocorona exhibits a transition from optically thick to optically thin around $3 R_{\mathrm{E}}$ (Østgaard et al., 2003) the model is split into 2 segments: Segment (A) is valid for $r=R / R_{\mathrm{E}}<3$ and Segment (B) is valid for $r=R / R_{\mathrm{E}} \geq 3$. The two segments provide at the interface distance at $r=$ $R / R_{\mathrm{E}}=3$ nearly the same H-density of $\approx 800 \mathrm{~cm}^{-3}$, so a smooth transition from one segment to the other takes place.

\subsection{Segment (A): below $3 R_{\mathrm{E}}$}

For geocentric distances $r=R / R_{\mathrm{E}}<3$ no TWINS1-LAD data are used to obtain the H-density distribution, because that optically thick region is not yet covered by our line of sight calculations, which assume optically thin conditions. For those distances we use a simply r-dependent Chamberlain (1963)-like H-density model (with a critical satellite altitude of $2.5 R_{\mathrm{E}}$ ). Carruthers et al. (1976) calculated Hdensities based on a Chamberlain (1963)-model for distances less than $3 R_{\mathrm{E}}$, which can be used and fitted into the following, simple approximation $\left(n_{\mathrm{H}}(r)\right.$ in $\mathrm{H}$-atoms per $\left.\mathrm{cm}^{-3}\right)$ :

$n_{\mathrm{H}}(r)=A \cdot \exp (B / r)$

We fitted the 2 coefficients to their best numerical values of $A=70.005204$ and $B=7.5497632$ for geocentric distances between $r \approx 1000 \mathrm{~km}$ and $r=R / R_{\mathrm{E}}<3$.
This approximated Chamberlain (1963)-like H-model delivers (assumed to be within $\approx 5-20 \%$ ) $\mathrm{H}$-densities from geocentric distances $r=R / R_{\mathrm{E}}<3$ down to $\approx 1000 \mathrm{~km}$ altitude over the Earth surface.

\subsection{Segment (B): above $3 R_{E}$}

\subsubsection{Mathematical model formulation}

For the outer neutral exosphere with $r=R / R_{\mathrm{E}} \geq 3$ the spherical harmonic representation of the three-dimensional $\mathrm{H}$ density is used:

$n_{\mathrm{H}}(r, \theta, \phi)=N(r) \sqrt{4 \pi} \sum_{l=0}^{2} \sum_{m=0}^{l} Z(r, \theta, \phi)$

with

$Z=\left[A_{\operatorname{lm}}(r) \cos (m \phi)+B_{\operatorname{lm}}(r) \sin (m \phi)\right] Y_{\operatorname{lm}}(\theta)$

where $Y_{\operatorname{lm}}(\theta)$ are the spherical harmonic Legendrepolynomials. This 12-coefficient parameterization is based on the simplified version of the Hodges (1994) model that was employed by Zoennchen et al. (2010).

Because this new model excludes seasonal and other timedependent effects, we used a maximum number of $l=2$ and neglected all the $B_{\operatorname{lm}}(r)$-coefficients to produce a sunaligned, $\phi$-symmetric structure for the exospheric $\mathrm{H}$ distribution as expected for an atmosphere under solar control. The $r$ (in $R_{\mathrm{E}}$ ), $\theta$, and $\phi$ values are given in GSE-coordinates.

Furthermore the in principle arbitrary $r$-dependences of the $A_{\operatorname{lm}}(r)$-coefficients were replaced by linear functions $A_{\mathrm{lm}}=\left(a_{\mathrm{lm}}+b_{\mathrm{lm}} \cdot r\right) \times 10^{-4}$ as suggested by fits done with the Hodges-model. To handle particularly the $\mathrm{N}(\mathrm{r})$-term a power law $N(r)=p \cdot r^{-k}$ function was used.

\subsubsection{Best model fit representation}

Including all selected TWINS1-LAD data of the named 4 time periods between June-September 2008 yields the following values for the 12 model coefficients:

$$
\begin{array}{lll}
N(r)=p \cdot r^{-k} & p=22364.7 ; & k=2.99318 \\
A_{00}(r)=1 & \text { always } & \\
A_{10}(r)=a_{10}+b_{10} \cdot r & a_{10}=-373.11 ; & b_{10}=178.20 \\
A_{11}(r)=a_{11}+b_{11} \cdot r & a_{11}=-1850.94 ; & b_{11}=509.44 \\
A_{20}(r)=a_{20}+b_{20} \cdot r & a_{20}=1592.13 ; & b_{20}=-309.11 \\
A_{21}(r)=a_{21}+b_{21} \cdot r & a_{21}=0 ; & b_{21}=0 \\
A_{22}(r)=a_{22}+b_{22} \cdot r & a_{22}=-1409.19 ; & b_{22}=361.05
\end{array}
$$

Note that this fit was performed with the assumption of isotropic Lyman- $\alpha$ resonant scattering.

Our H-density profiles of both model segments (A) and (B) are presented for the day and the night side separately in Fig. 4 in comparison with the yearly average H-density profile (for solar-zenith-angle $90^{\circ}$ ) from Østgaard et al. (2003) 


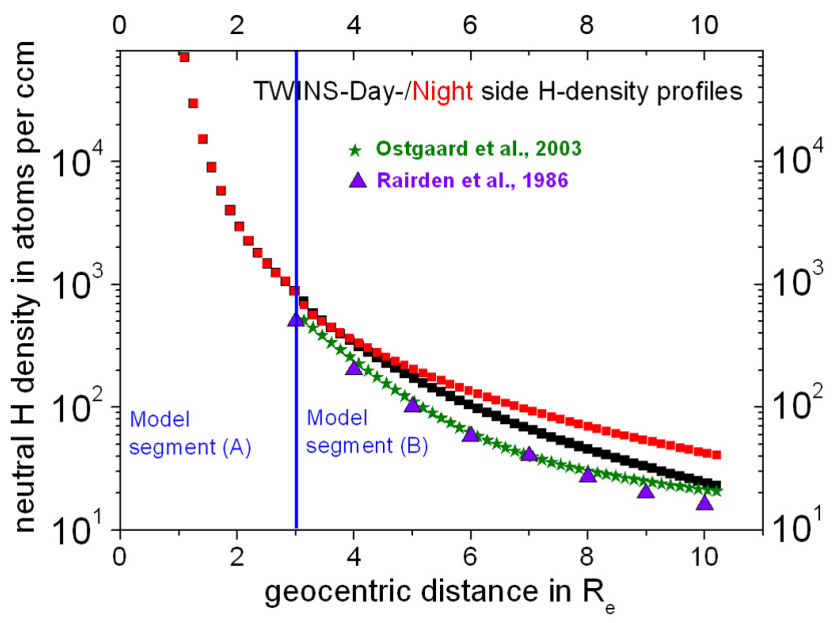

Fig. 4. Dayside $\left(\phi=0^{\circ}\right.$, black $) /$ nightside $\left(\phi=180^{\circ}\right.$, red $)$ profiles of the TWINS H-density model in comparison with the yearly average $\phi=90^{\circ} \mathrm{H}$-density profiles from Østgaard et al. (2003) (green stars) and from Rairden et al. (1986) (violet triangles).

and Rairden et al. (1986). Figure 5 shows the H-density contours of the geocentric ecliptic equatorial and the geocentric ecliptic meridional (polar) cross section of our model.

\section{Regime of validity (distance, solar activity, time)}

\subsection{Valid distances: from $\approx 1000 \mathrm{~km}$ altitude to geocen- tric distance $8 R_{\mathrm{E}}$}

The fit was done using TWINS lines-of-sight with geocentric intersection distances larger than $3 R_{\mathrm{E}}$, for the reason to be able to use the optically thin approximation. A Chamberlainlike density model for the lower regions matches quite well at the interface distance $3 R_{\mathrm{E}}$ with the TWINS data fit and will be therefore implemented below $3 R_{\mathrm{E}}$ for altitudes down to $1000 \mathrm{~km}$.

\subsection{Valid solar conditions: quiet, solar minimum condi- tions $\left(F_{10.7 \mathrm{~cm}}<100\right)$}

The fit was done using data from the solar quiet minimum with $F_{10.7 \mathrm{~cm}} \approx 68 \pm 2.5 \%\left[10^{-22} \mathrm{~W} \mathrm{~m}^{-2} \mathrm{~Hz}^{-1}\right]$ between 11 June and 13 September 2008 and should be used only in situations with comparable solar activity.

\subsection{Valid time frame of use: between June 2008 until early 2010}

Low solar activity prevailed through early 2010 , with solar ultraviolet fluxes that were comparable to period JuneSeptember 2008 for which LAD data were gathered.
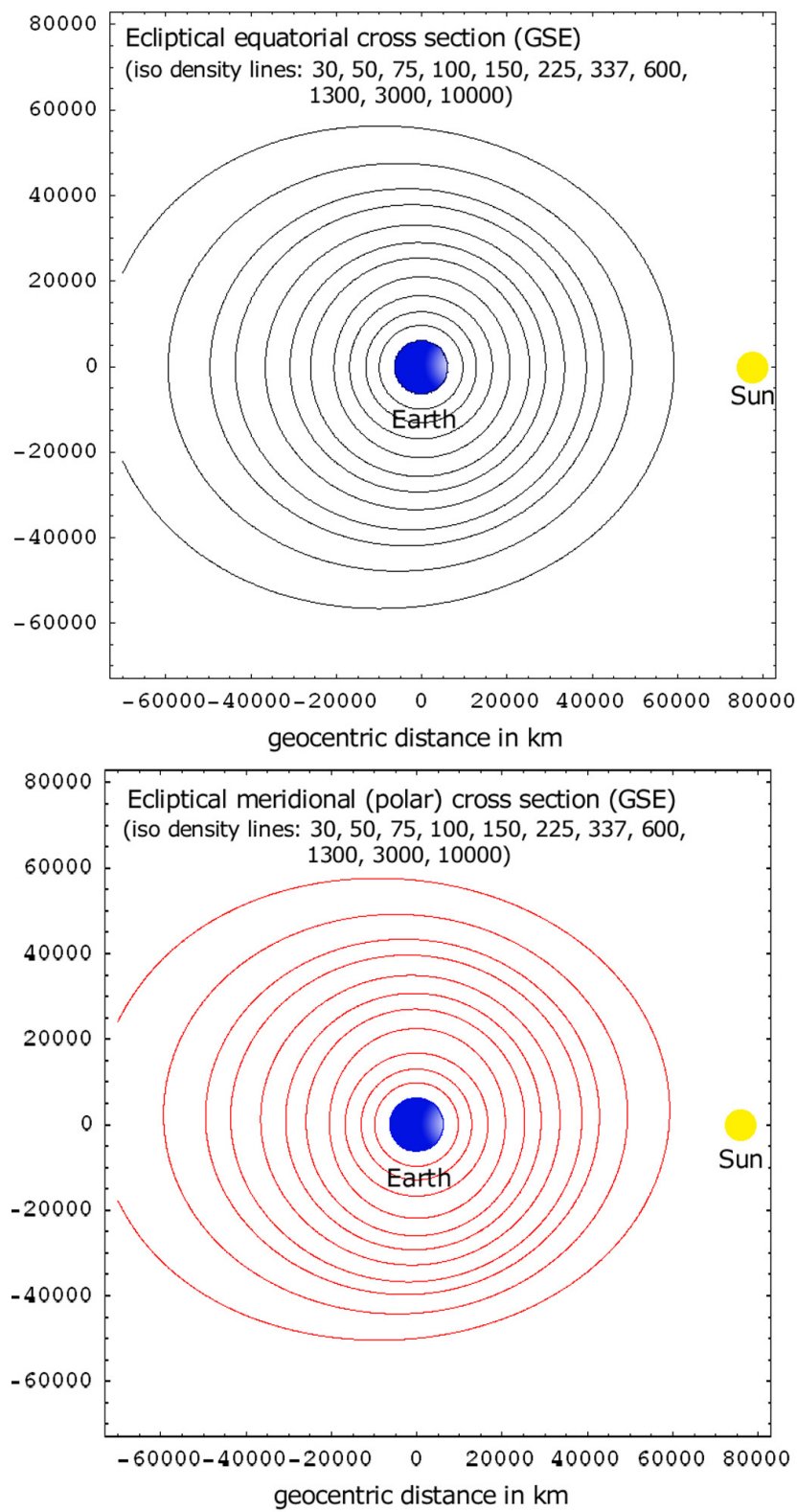

Fig. 5. Ecliptic equatorial and -meridional cross sections of the $\mathrm{H}-$ density model.

\section{Day of year usability}

In order to get the neutral H-density for a vector given in an user-defined coordinate system $\boldsymbol{K}$ on a particular day, this $\boldsymbol{K}$-vector needs simply to be converted in GSE-coordinates for that day before calling the model function $n_{\mathrm{H}}(r, \theta, \phi)$ using the presented coefficients. This delivers trustable results (with errors given below) during the time period June 2008 through early 2010 (as mentioned above, owing to the nearly constant solar minimum conditions). 

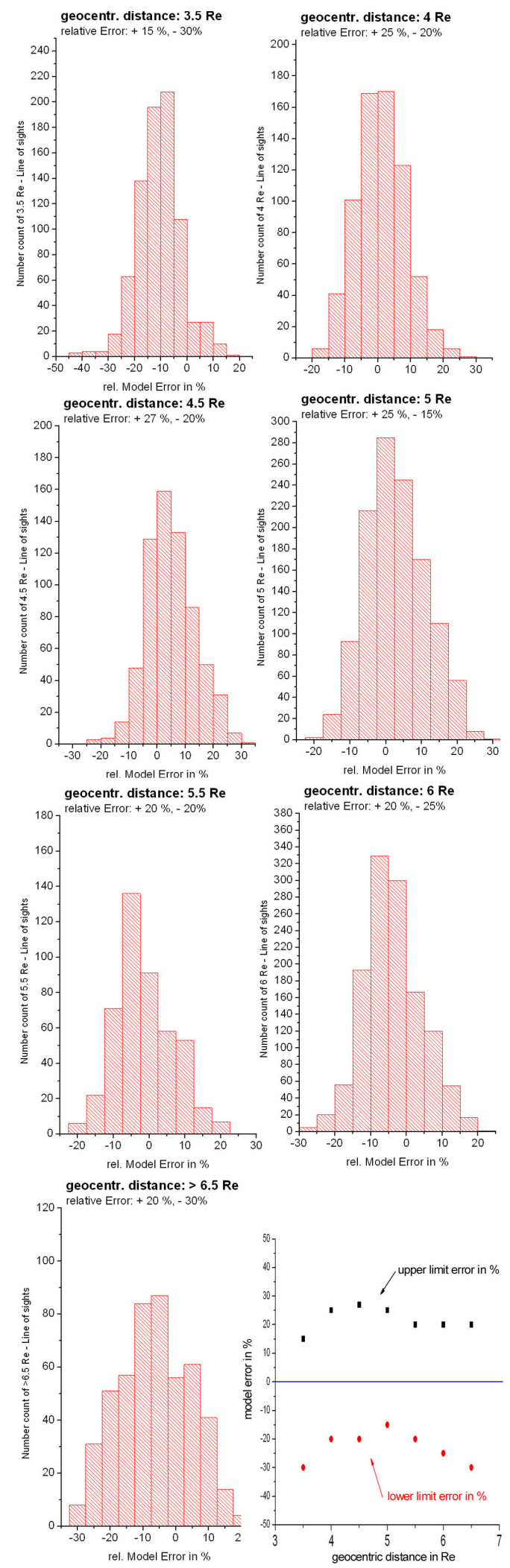

Fig. 6. Relative error histograms for different geocentric distances; the relative max./min. errors in the caption of each figure are the borders of the $2 \sigma$-interval of a Gaussian fit.

\section{Errors}

Since the Lyman $\alpha$ intensitiy $I$ of a line-of-sight (LOS) has its main contribution from neutral hydrogen atoms in the region of the LOS's Earth intersection distance (E.i.d.) the relative hydrogen density error at this distance is (more or less) the same as the relative error of the Ly- $\alpha$ intensity reconstructed from the model $I_{\text {Model }}$ compared with the TWINSmeasured Ly- $\alpha$ intensity $I_{\text {Data }}$.

The relative hydrogen density error at a distance $r_{0}$ using a line-of-sight (LOS) with the same Earth intersection distance $r_{\text {E.i.d. }}=r_{0}$ is therefore representable as:

$\Delta n_{\mathrm{H}}\left(r_{0}=r_{\text {E.i.d. }}\right) \approx \Delta I_{\mathrm{LOS}}\left(r_{\text {E.i.d. }}\right)$

$\approx\left(I\left(r_{\text {E.i.d. }}\right)_{\text {Model }}-I\left(r_{\text {E.i.d. }}\right)_{\text {Data }}\right) / I\left(r_{\text {E.i.d. }}\right)_{\text {Data }}$

Figure 6 shows the relative hydrogen density errors obtained from the relative error between the model intensities and the measured intensities for geocentric distances between 3-7 $R_{\mathrm{E}}$. The borders of the percentage error ranges (given in percent) as written in Fig. 6 are calculated as the width of the $2 \sigma$-interval of a Gaussian fit for each plot in Fig. 6 (i.e. $\approx 95 \%$ of the LOS-errors are inside the percentage error range).

The estimated uncertainty in the obtained $\mathrm{H}$ density distribution is nearly constant at $15 \%$ to $20 \%$ from 3 to $7 R_{\mathrm{E}}$ but would then rapidly increase up to a factor of 2 at $10 R_{\mathrm{E}}$. For lower geocentric distances $r<3 R_{\mathrm{E}}$ (within the region of the Chamberlain-like model) we assume a relative error of $\approx 5-20 \%$.

Above $8 R_{\mathrm{E}}$ the errors are $\approx \pm 30 \%$ and grow larger with increasing distance, to a value at $10 R_{\mathrm{E}}$, which might be $100 \%$. So we recommend that use of the model be restricted to $r=R / R_{\mathrm{E}} \leq 8$, or used with appropriate caution and error analysis outside this range.

\section{Conclusions}

LAD measurements from TWINS-1 during the solar minimum conditions between June and September 2008 have been used to obtain a global exospheric H-density distribution for geocentric distances from 3 to $8 R_{\mathrm{E}}$. For lower geocentric distances, a best fitting r-dependent Chamberlain (1963)-like H-density model has been adapted. The radial dependence of our spherically symmetric distribution, $N(r)=p \cdot r^{-k}$, is in agreement with the density profiles obtained by Rairden et al. (1986) and Østgaard et al. (2003). Our global distribution is essentially cylindrically symmetric about the Sun-Earth line, exhibiting an enhancement in the antisolar direction that is consistent with the location of a geotail. Such asymmetry is in general agreement with the findings of Østgaard et al. (2003) or Carruthers et al. (1976). 
Acknowledgements. The authors gratefully thank the TWINS team (PI Dave McComas) for making this work possible. We also thank Wayne Pryor and Randy Gladstone for providing representative interplanetary glow maps.

J. H. Zoennchen, U. Nass and H. J. Fahr acknowledge the support by the DLR grant FKZ 50 OE 0901.

J. J. Bailey and M. Gruntman acknowledge support by the NASA TWINS mission; J. J. Bailey also acknowledges support by a Northrop Grumman fellowship.

Topical Editor C. Jacobi thanks W. Pryor and another anonymous referee for their help in evaluating this paper.

\section{References}

Bailey, J. and Gruntman, M.: Experimental study of exospheric hydrogen atom distributions by Lyman- $\alpha$ detectors on the TWINS mission, J. Geophys. Res., 116, A09302, doi:10.1029/2011JA016531, 2011.

Bertaux, J. L., Quémerais, E., Lallement, R., Kyrölä, E., Schmidt, W., Summanen, T., Goutail, J. P., Berthé, M., Costa, J., and Holzer, T.: First Results from SWAN Lyman $\alpha$ solar wind mapper on SOHO, Sol. Phys., 175, 737, doi:10.1023/A:1004979605559, 1997.

Bishop, J.: Analytic exosphere models for geocoronal application, Planet. Space Sci., 39, 885-893, 1991.

Carruthers, G. R., Page, T., and Meier, R. R.: Apollo 16 Lyman alpha imagery of the hydrogen geocorona, J. Geophys. Res., 81, 1664-1672, 1976.

Chamberlain, J. W.: Planetary coronae and atmospheric evaporation, Planet. Space Sci., 11, 901-960, 1963.

Fahr, H. J. and Shizgal, B.: Modern Exospheric Theories and Their Observational Relevance, Rev. Geophys. Space Phys., 21, 75124, 1983.

Grimes, E. W., Perez, J. D., Goldstein, J., McComas, D. J., and Valek, P.: Global observations of ring current dynamics during CIR-driven geomagnetic storms in 2008, J. Geophys. Res., 115, A11207, doi:10.1029/2010JA015409, 2010.

Hodges Jr., R. R.: Monte Carlo simulation of the terrestrial hydrogen exosphere, J. Geophys. Res., 99, 23229-23247, 1994.

Johnson, F. S.: The Distribution of Hydrogen in the Telluric Hydrogen Corona, Astrophys. J., 133, 701, 1961.

Kupperian, J. E., Byram, E. T., Chubb, T. A., and Friedman, H.: Far ultra-violet radiation in the night sky, Planet. Space Sci., 1, 3, doi:10.1016/0032-0633(59)90015-7, 1959.

McComas, D. J., Allegrini, F., Baldonado, J., Blake, B., Brandt, P. C., Burch, J., Clemmons, J., Crain, W., Delapp, D., Demajistre, R., Everett, D., Fahr, H., Friesen, L., Funsten, H., Goldstein, J., Gruntman, M., Harbaugh, R., Harper, R., Henkel, H., Holmlund, C., Lay, G., Mabry, D., Mitchell, D., Nass, U., Pollock, C., Pope, S., Reno, M., Ritzau, S., Roelof, E., Scime, E., Sivjee, M., Skoug, R., Sotirelis, T. S., Thomsen, M., Urdiales, C., Valek, P., Viherkanto, K., Weidner, S., Ylikorpi, T., Young, M., and Zoennchen, J.: The two wide-angle imaging neutral-atom spectrometers (TWINS)NASA mission-of-opportunity, Space Sci. Rev., 142, 157, doi:10.1007/s11214-008-9467-4, 2009.
Nass, H. U., Zoennchen, J. H., Lay, G., and Fahr, H. J.: The TWINS-LAD mission: Observations of terrestrial Lyman- $\alpha$ fluxes, ASTRA Astrophysics and Space Sciences Transactions, 2, 27-31, 2006.

Østgaard, N., Mende, S. B., Frey, H. U., Gladstone, G. R., and Lauche, H.: Neutral hydrogen density profiles derived from geocoronal imaging, J. Geophys. Res. Space Phys., 108, 1300, doi:10.1029/2002JA009749, 2003.

Pryor, W. R., Ajello, J. M., Barth, C. A., Hord, C. W., Stewart, A. I. F., Simmons, K. E., McClintock, W. E., Sandel, B. R., and Shemansky, D. E.: The Galileo and Pioneer Venus Ultraviolet Spectrometer Experiments: Solar Lyman-a Latitude Variation at Solar Maximum from Interplanetary Lyman- $\alpha$ Observations, Astrophys. J., 394, 363-377, 1992.

Pryor, W. R., Lasica, S. J., Stewart, A. I. F., Hall, D. T., Lineaweaver, S., Colwell, W. B., Ajello, J. M., White, O. R., and Tobiska, W. K.: Interplanetary Lyman a observations from Pioneer Venus over a solar cycle from 1978 to 1992, J. Geophys. Res., 103, 26833-26849, 1998.

Pryor, W., Gangopadhyay, P., Sandel, B., Forrester, T., Quemerais, E., Moebius, E., Esposito, L., Stewart, I., McClintock, B., Jouchoux, A., Colwell, J., Izmodenov, V., Malama, Y., Tobiska, K., Shemansky, D., Ajello, J., Hansen, C., Bzowski, M., and Frisch, P.: Radiation transport of heliospheric Lyman- $\alpha$ from combined Cassini and Voyager data sets, Astron. Astrophys., 491, 21-28, 2008.

Rairden, R. L., Frank, L. A., and Craven, J. D.: Geocoronal imaging with Dynamics Explorer, J. Geophys. Res., 91, 13613-13630, 1986.

Thomas, G. E. and Bohlin, R. C.: Lyman-alpha measurements of neutral hydrogen in the outer geocorona and in interplanetary space, J. Geophys. Res., 77, 2752-2761, 1972.

Woods, T. N., Tobiska, W. K., Rottman, G. J., and Worden, J. R.: Improved solar Lyman alpha irradiance modeling from 1947 through 1999 based on UARS observations, J. Geophys. Res., 105, 27195-27215, doi:10.1029/2000JA000051, 2000.

Zoennchen, J. H.: Modellierung der dreidimensionalen Dichteverteilung des geokoronalen Neutralwasserstoffes auf Basis von TWINS Ly-Alpha-Intensitaetsmessungen, Phd thesis, URN: urn:nbn:de:hbz:5N-08886, URL: http://www.ulb.uni-bonn.de/, 2006.

Zoennchen, J. H., Nass, U., Lay, G., and Fahr, H. J.: 3-Dgeocoronal hydrogen density derived from TWINS Ly- $\alpha$-data, Ann. Geophys., 28, 1221-1228, doi:10.5194/angeo-28-12212010, 2010. 